\title{
MODELO DE AUTOMATIZAÇÃO DO CALIBRADOR FLUKE MODELO $5720 A$ E DO MULITÍMETRO DIGITAL HEWLETT PACKARD 3458A
}

\author{
Lucas Rabelo de Oliveira \\ Graduando em Engenharia Elétrica pelo Centro Universitário Augusto Motta (UNISUAM) \\ Rio de Janeiro, RJ, Brasil \\ lucasrabelo194@outlook.com \\ Antônio José Dias da Silva \\ Mestre em Sistemas de Informação pela Universidade Federal do Rio de Janeiro (UFRJ) \\ Professor da Faculdade Cesgranrio - FACESG \\ Rio de Janeiro, RJ, Brasil \\ antoniojose@cesgranrio.org.br \\ Geraldo Motta Azevedo Junior \\ Doutor em Engenharia Elétrica pela Universidade Federal do Rio de Janeiro (COPPE/UFRJ) \\ Professor do Centro Universitário Augusto Motta - UNISUAM \\ Rio de Janeiro, RJ, Brasil \\ geraldomotta@unisuam.edu.br \\ André Luis da Silva Pinheiro \\ Doutor em Engenharia Nuclear pela Universidade Federal do Rio de Janeiro (UFRJ) \\ Professor do Centro Universitário Augusto Motta - UNISUAM \\ Rio de Janeiro, RJ, Brasil \\ apinheiro99@gmail.com
}

\section{RESUMO}

Sabe-se que na Engenharia, o uso de instrumentos capazes de realizar medições corretas é de suma importância. Logo o objetivo deste estudo é propor um método de calibração automatizada para o calibrador fluke modelo $5720 \mathrm{~A}$ e do multímetro digital hewlett packard 3458a, ambos usados em medições de correntes e tensões elétricas. Para isso foi usado a interface GPIB do padrão de referência no instrumento sob calibração e o software LabView. Como resultado, após coleta dos valores aferidos, gerou-se uma planilha comparativa com calibrações validadas de períodos passados. O método proposto mostrou maior otimização do tempo de calibração, melhora da qualidade e segurança dos resultados, tornando-o mais eficiente.

Palavras-chave: Automatização, Calibração, CEPEL, Multímetro, LabVIEW.

\begin{abstract}
It is known that in Engineering, the use of instruments capable of making correct measurements is extremely important. Therefore, the objective of this study is to propose an automated calibration method for the fluke calibrator model 5720A and the hewlett packard 3458 digital multimeter, both used in current and voltage measurements. For this, the reference standard GPIB interface was used on the instrument under calibration and the LabView software. As a result, after collecting the measured values, a comparative spreadsheet with validated calibrations of past
\end{abstract}


periods was generated. The proposed method showed greater optimization of the calibration time, improvement of the quality and safety of the results, making it more efficient.

KeyWords: Automation, Calibration, CEPEL, Multimeter, LabVIEW.

\section{INTRODUÇÃO}

Nos diversos cursos de engenharia, ao longo de investigações e acontecimentos físicos, empenha-se frequentemente com variáveis, apresentadas por meio de valores de observações e medições, Dessa forma, é importante falar a respeito do processo de calibração instrumentos elétricos. Sendo assim, é de imensa importância que as possíveis variáveis apresentem seus valores incisivos na melhor resolução possível, ou seja, melhores valores de medições expressos, visto que, pelo contrário, existirá desenvolvimento de erro.

Desta forma, a área de calibração de instrumentos elétricos foi criada com a finalidade de certificar a confiabilidade de instrumentos elétricos de tal forma a observar as fundamentais características metrológicas de sinais elétricos, de acordo com referências do INMETRO. Um laboratório de metrologia eficaz é uma fatia importante para qualquer indústria que tenha o objetivo de produzir com qualidade adequada. (COUTO e MONTEIRO, 2000).

Além disso, o processo de calibração é responsável por calibrar os instrumentos e analisar as melhores apresentações das medidas, objetivando sempre definir a melhor qualidade das medições, estes instrumentos necessitam atravessar frequentemente por metodologias de calibração, fundamentalmente, a calibração é definida pelo meio de exames dos valores mostrados ao longo de instrumento verificador em confrontação com o modelo padrão equivalente (INMETRO, 1999);. No entanto, será necessário assegurar que os métodos de calibração acompanhem também uma sequência de regra, a fim de que se mantenha a qualidade e uniformidade. Tendo assim esse propósito à norma NBR ISO/IEC 17025:2005: controlar a eficácia das metodologias e padronizar, desta forma garantindo a capacidade técnica dos serviços.

Sendo assim, o principal objetivo foi propor uma método de calibração automatizada visando desenvolver o processo de calibração do multímetro Hewlett Packard modelo 3458A, sendo calibrador pelo calibrador padrão Fluke modelo 5720A, abrangendo as escalas de tensão alternada e tensão contínua.

\section{DESENVOLVIMENTO}




\subsection{Prudências em uma Calibração Automatizada}

Como foi citado anteriormente, a metodologia de calibração de instrumento deve manter sempre o nível de confiabilidade dos métodos de aplicação da calibração, visando sempre melhorar a incerteza de medição.

Assim sendo, deve se analisar todo o processo com prudência, deste modo a metodologia de automatização de calibrações de instrumentos tem sido cada vez mais requisitada, porque a autocalibração tem gerado melhor desempenho na demanda e qualidade de serviços para os laboratórios de calibrações, trazendo benefícios aos técnicos de calibração no modo de execução, porém se deverá agir com prudência.

Um projeto de automatização de calibradores e multímetros, será determinante na otimização do tempo e qualidade do laboratório, pois estabelece mais exatidão nas medições, proteção ao técnico e ao instrumento, pois o próprio instrumento realizará as configurações das medidas.

Para a segurança da metodologia de automatização do calibrador Fluke modelo 5720A e do multímetro digital Hewlett Packard modelo 3458A, é importante que para o devido funcionamento do projeto, instalações, cabos, estabilização entre outros fatores, sejam examinados e aplicados nas funções devidas de cada item, por profissionais certificados e capacitados, para evitar deste modo, possíveis acidentes e colocar a vida de outros funcionários do local em risco. Porque em uma instalação mal feita, poderá acarretar sérios problemas, desde a perda total dos instrumentos curtos circuitos no sistema, até danos maiores para o laboratório.

Dessa forma, o laboratório deve seguir todos os procedimentos segundo a norma, NBR IEC 17025:2005, "requisitos gerais para a competência de laboratórios de Ensaios e calibração" combina as determinações das normas ISO 9001, 9002, no que diz respeito ao gerenciamento e garantia de qualidade, e do ISO/IEC guia 25 , no que se referia às exigências técnicas em laboratórios, (DINIZ e MULLER, 2007), é necessário seguir os procedimentos e regulações da norma NBR ISO/IEC 17025 da Associação Brasileira de Normas Técnicas (ABNT), requisitos gerais para competência de laboratórios de calibração e ensaio. Na Figura 1 mostra os procedimentos gerais que nos quais o laboratório deve seguir na metodologia do sistema de calibração. 
Figura 1 - Procedimentos que o laboratório deve seguir no processo de calibração

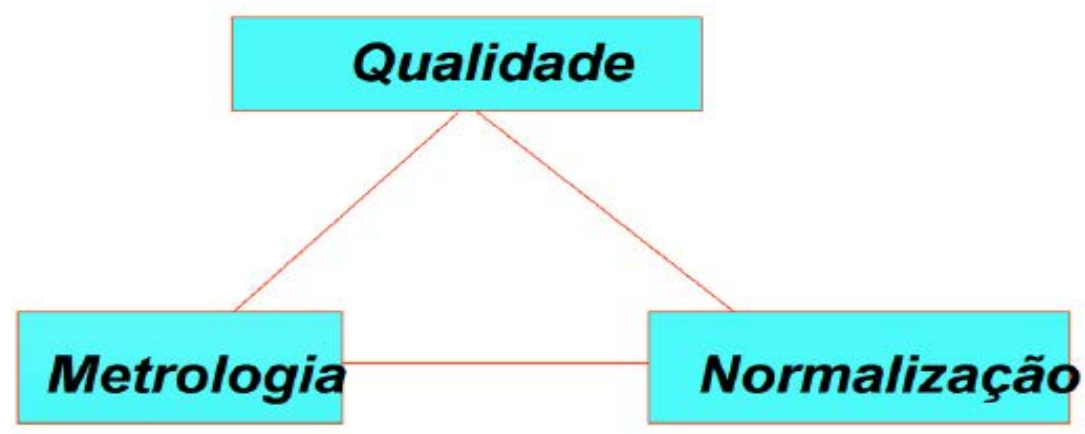

Fonte: INMETRO (200)

\subsection{Autocalibração e Medição}

Assim para aplicação do Multímetro Hewlett packard 3458A e o Calibrador Fluke modelo 5720A, será preciso à utilização de sua metodologia de autocalibração. De certa forma para omitir as fontes de incerteza correlacionadas às tensões residuais e às variações de temperatura maiores que $5 \stackrel{\circ}{\circ}$, que de certo modo acarretarão em possíveis influencias significativas no cálculo de incerteza de medição. Neste método necessitam ser feitos conforme as devidas periodicidades de comandos, para este padrão de trabalho necessitam que, o Calibrador Fluke Modelo 5720A utilize o Multímetro Hewlett Packard Modelo 3458A como padrão de referência, sendo assim respeitando o procedimento. Estabilização térmica (warm-up), autocalibração e autozero dos instrumentos envolvidos na calibração devem estar de acordo com o manual técnico dos instrumentos. Calibrador Fluke Modelo 5720A - Periodicidade: Mensal. Comandos SETUP MENUS $>$ CAL > ZERO. Multímetro Hewlett Packard Modelo 3458A - Periodicidade: Mensal. Comandos: $\mathrm{CAL}>\mathrm{AUTO}$

Necessita-se conectar a interface GPIB do padrão de referência no instrumento sob calibração e o software LabView, sendo este responsável pela conexão e comunicação entre o computador, o multímetro Hewlett Packard modelo 3458A e o calibrador padrão fluke modelo 5720A, escalas de tensão contínua e alternada, no qual autoriza o processamento de sinais em tempo real com incorporação de hardware no computador, facilitando o técnico responsável na metodologia de calibração automatizada, logo após conectar ao microcomputador que será utilizado, como mostra o circuito geral para aplicação da calibração automatizada do multímetro Figura 2. 
Figura 2 - Circuito para autocalibração

Fonte: Autores

Em seguida, a metodologia é aplicada no computador do laboratório e do software LabVIEW, mostra o estado inicial do programa em interconexão com o calibrador e o multímetro, onde apresenta a Figura 3 a aba de configuração inicial do programa sendo direcionada para os endereços devidos, no caso o calibrador fluke modelo 5720A e o multímetro hewlett packard 3458A sendo direcionados para o cabo GPIB. No estado inicial, na parte superior existem três abas, sendo estas, configuração inicial, parâmetros e medição. Na aba de "configuração inicial", têm por finalidade definir o que será utilizado na calibração, endereço do calibrador no caso o calibrador fluke modelo 5720A, o endereço do medidor o multímetro Hewlett Packard e o modelo do medidor 3458A. Em seguida apresenta se uma caixa que tem por função direcionar e salvar na planilha de medição para o cálculo de incerteza os valores conquistados.

$\mathrm{Na}$ parte de status do padrão e status do medidor tem finalidade de mostrar se os padrões estão em funcionamento ou não (ligado ou desligado), exibindo os padrões que estão ligados ao software.

Na segunda aba "Parâmetros" mostra exemplos das grandezas a serem afeiçoadas. Em seguida na aba de "Medição" apresenta as caixas nos pontos que serão calibrados, no caso os três pontos de tensão, a frequência, número de medições, tempo de estabilização e número de pontos. 
Figura 3 - Configuração de parâmetros

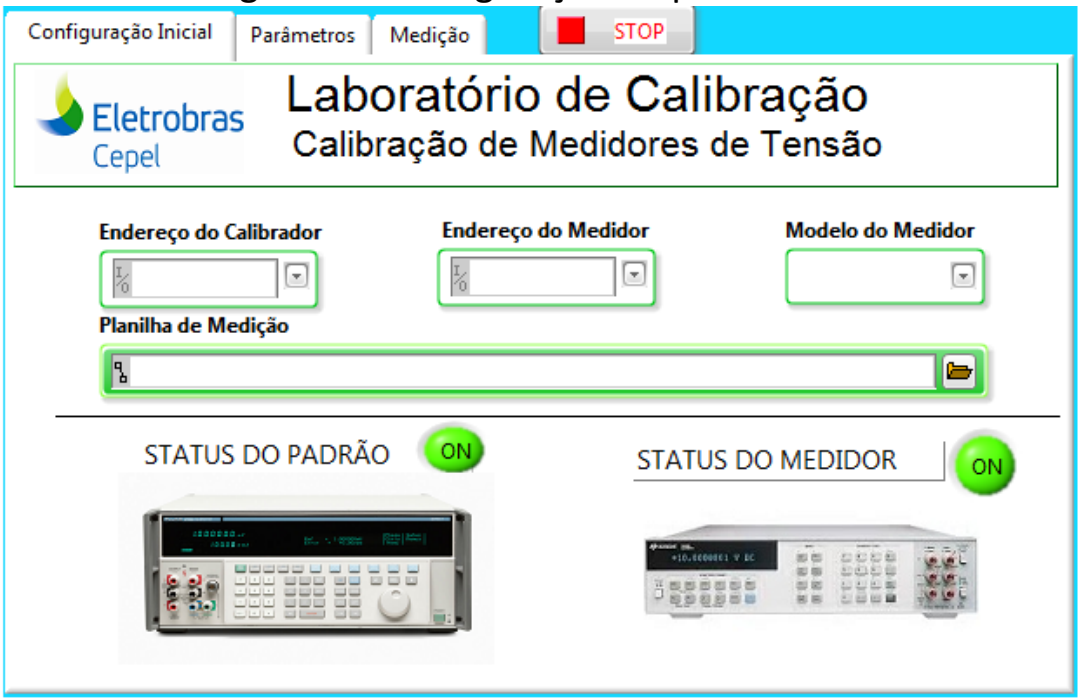

Fonte: Autores

\subsection{Análises dos Resultados}

Os valores que forem medidos, verificados na calibração serão diretamente registrados na planilha que foi selecionada, como mostra a Figura 4.

Figura 4 - Valores obtidos na calibração automatizada

\begin{tabular}{|c|c|c|c|}
\hline Medidas: & 9,99887 & 14,9952 & 19,9927 \\
\hline & 9,99889 & 14,9953 & 19,9926 \\
\hline & 9,99886 & 14,9953 & 19,9924 \\
\hline
\end{tabular}

Fonte: Autores

Deste modo por demonstração, obteve resultado melhorado em comparação técnica com calibrações validadas de períodos passados como mostra a Figura 5.

Figura 5 - Comparação dos Resultados

\begin{tabular}{|c|c|c|c|c|}
\multicolumn{1}{c}{$\begin{array}{c}\text { Resultados } \\
\text { Valor de } \\
\text { Referência }\end{array}$} & $\begin{array}{c}\text { Valor } \\
\text { Medido }\end{array}$ & Desvio & Incerteza & Fator k \\
\hline 10,000 & 9,999 & $-0,01$ & 0,080 & 2,00 \\
\hline 15,00 & 15,00 & 0,00 & 0,080 & 2,00 \\
\hline 20,00 & 19,99 & $-0,05$ & 0,080 & 2,00 \\
\hline
\end{tabular}

\begin{tabular}{|c|c|c|c|c|}
\hline $\begin{array}{l}\text { Valor de } \\
\text { Referência }\end{array}$ & $\begin{array}{c}\text { Resultados } \\
\text { Valor } \\
\text { Medido } \\
\end{array}$ & Desvio & Incerteza & Fator $\mathrm{k}$ \\
\hline 10,000 & 10,000 & 0,00 & 0,07 & 2,00 \\
\hline 15,00 & 15,00 & 0,00 & 0,07 & 2,00 \\
\hline 20,00 & 20,00 & 0,00 & 0,07 & 2,00 \\
\hline
\end{tabular}

Fonte: Autores 
Nota-se que na maior parte dos resultados das incertezas se baseia na CMC (Capacidade de Medição e Calibração), que (INMETRO, 2003) é a menor incerteza de medição que o laboratório é capaz de adquirir, aplicando dentro do seu escopo de acreditação. Neste caso do laboratório CA1, o nível de confiabilidade e a probabilidade de abrangência das medições são de mais ou menos $95 \%$.

Sendo assim a mostra Tabela 1, cada valor de CMC (Capacidade de Medição e Calibração), para cada escala e faixa de grandeza de medição que são aplicadas na calibração do multímetro. Que se refere ao DLA (Departamento de Laboratórios de Adrianópolis), Pela RBC (Rede Brasileira de Calibração), tem acreditação e número de identificação 0008.

Tabela 1 - Escopo de Acreditação do laboratório CA1

\begin{tabular}{|c|c|c|}
\hline Descrição do Serviço & Faixa & CMC \\
\hline \multirow{3}{*}{ Medidor de Tensão AC } & $10 \mathrm{mV}$ até $650 \mathrm{~V}(60 \mathrm{~Hz})$ & $0,07 \%$ \\
\cline { 2 - 3 } & $10 \mathrm{mV}$ até $650 \mathrm{~V}(1 \mathrm{kHz})$ & $0,07 \%$ \\
\cline { 2 - 3 } & $\begin{array}{c}10 \mathrm{mV} \text { até } 650 \mathrm{~V}(10 \\
\mathrm{kHz})\end{array}$ & $0,07 \%$ \\
\hline Medidor de Tensão DC & $20 \mathrm{mV}$ até $1000 \mathrm{~V}$ & $0,003 \%$ \\
\hline \multirow{3}{*}{ Medidor de Corrente AC } & $20 \mu \mathrm{A}$ até $<1 \mathrm{~A}(60 \mathrm{~Hz})$ & $0,06 \%$ \\
\cline { 2 - 3 } & $1 \mathrm{~A}$ até $10 \mathrm{~A}(60 \mathrm{~Hz})$ & $0,8 \%$ \\
\cline { 2 - 3 } & $20 \mu \mathrm{A}$ até $<1 \mathrm{~A}(1 \mathrm{kHz})$ & $0,06 \%$ \\
\cline { 2 - 3 } & $1 \mathrm{~A}$ até $10 \mathrm{~A}(1 \mathrm{kHz})$ & $0,11 \%$ \\
\hline Medidor de Corrente DC & $20 \mu \mathrm{A}$ até $<1 \mathrm{~A}$ & $0,08 \%$ \\
\cline { 2 - 3 } & $1 \mathrm{~A}$ até $9,5 \mathrm{~A}$ & $0,06 \%$ \\
\hline
\end{tabular}

Fonte: Adaptado INMETRO (2016)

Desta maneira, as maiores partes das incertezas de medições estabelecem seu valor na CMC conforme o resultado real ser uma incerteza menor, deste modo à macro (planilha de cálculo de incerteza) aproxima a incerteza final para a CMC. Isto procede, pois é um trabalho acreditação, sendo assim o laboratório de calibração não pode apresentar uma incerteza de medição menor que a sua $C M C$.

Nos dias atuais o laboratório de calibração CA1 apresenta uma melhor aparelhagem melhores instrumentos de medições para as calibrações, facilitando nas aplicações das metodologias e melhorando sua incerteza de medição chegando ao CMC, visando que o cálculo da CMC foi realizado no ano de 2000, desta forma a maior parte das incertezas obtidas nesta calibração apresenta melhor capacidade de medição CMC que há anterior. 
Com base nas calibrações realizadas manualmente e automatizada, através da Figura 6 percebe se uma melhora na otimização do tempo e compara os tempos médios da calibração automática e manual.

Figura 6 - Comparação do tempo estimado das calibrações

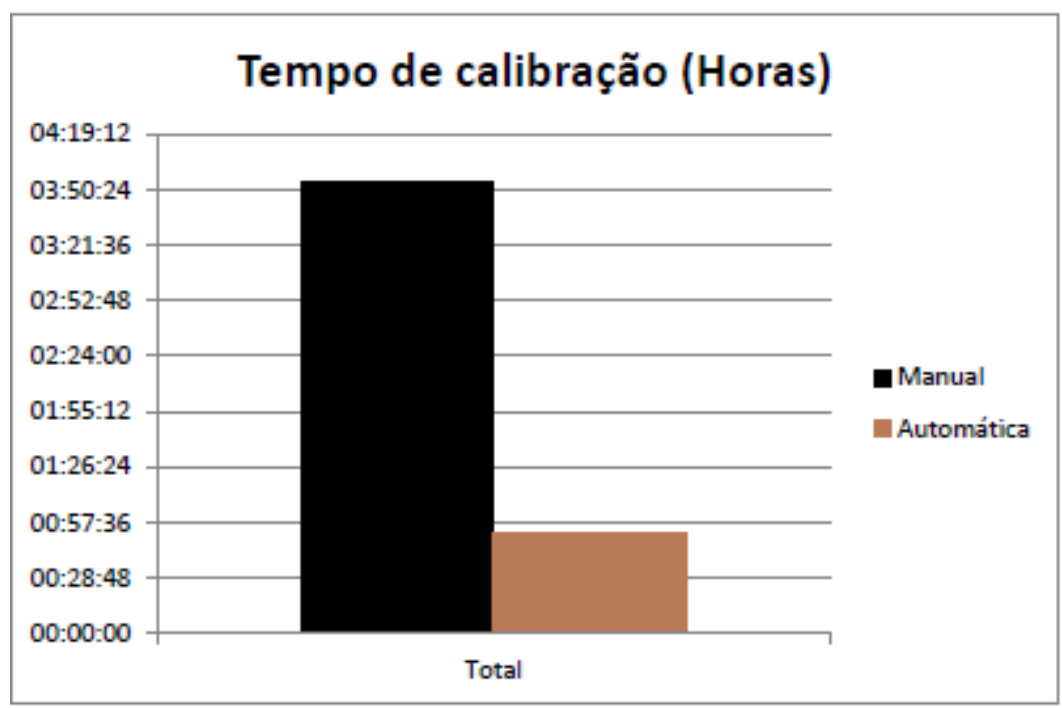

Fonte: Autores

Nesta análise gráfica percebe-se que o tempo da calibração automática para a calibração manual melhorou, desta forma teve uma redução aproximadamente de $80 \%$ em relação à calibração manual.

Sendo assim, as grandezas analisadas neste projeto foram nas escalas de tensão contínua e alternada, desta maneira obteve resultados de redução oscilando entre $69 \%$ a $81 \%$. Conforme o gráfico pode ser analisado as escalas de tensão na Figura 7.

Figura 7 - Tempo simulado de calibração nas escalas de tensões

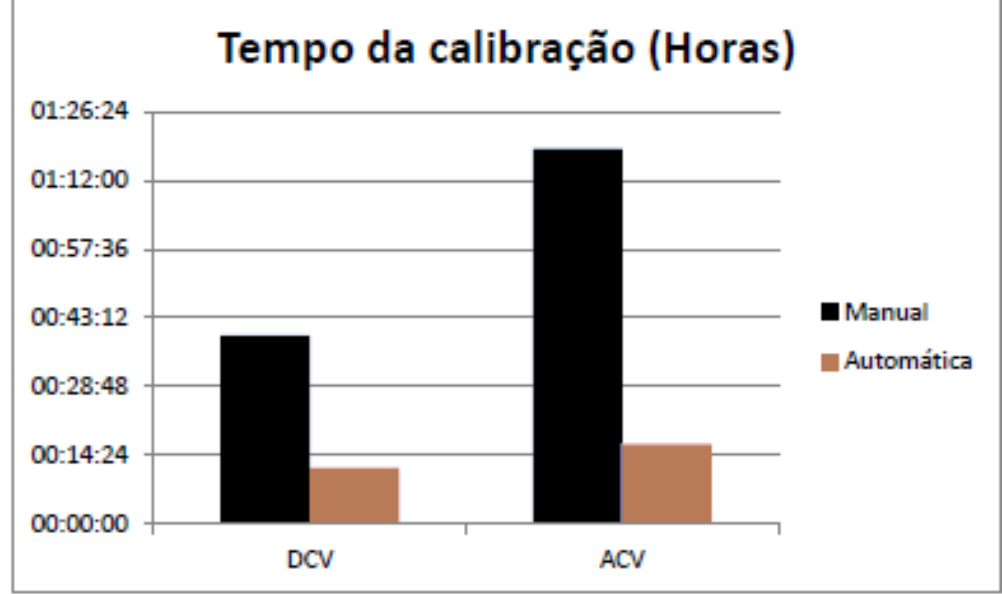

Fonte: Autores 
Na Tabela 2 é apresentado o tempo de realização das calibrações nas grandezas de tensão alternada e continua, para a tensão alternada deve-se levar em conta a frequência.

Tabela 2 - Tempo estimado dos métodos aplicados na calibração

\begin{tabular}{|c|c|c|c|}
\hline Grandezas & $\begin{array}{l}\text { Tempo de } \\
\text { calibração } \\
\text { automática }\end{array}$ & $\begin{array}{c}\text { Tempo de } \\
\text { calibração manual }\end{array}$ & $\begin{array}{c}\text { Redução } \\
\text { (\%) }\end{array}$ \\
\hline Escalas VAC $-60 \mathrm{~Hz}$ & $8 \min 27 s$ & $28 \min 36 s$ & 70,45 \\
\hline Escalas VAC - $1 \mathrm{KHz}$ & $5 \mathrm{~min} 45 \mathrm{~s}$ & $25 \min 43 \mathrm{~s}$ & 77,64 \\
\hline Escalas VAC - $10 \mathrm{KHz}$ & $4 \min 12 \mathrm{~s}$ & $22 \min 27 \mathrm{~s}$ & 81,29 \\
\hline Escalas VDC & $10 \min 31 \mathrm{~s}$ & $37 \mathrm{~min} 29 \mathrm{~s}$ & 71,94 \\
\hline Preencher cabeçalho & & $6 \mathrm{~min} 54 \mathrm{~s}$ & \\
\hline Total & $28 \min 55 \mathrm{~s}$ & $2 \mathrm{~h} 1 \min 9 \mathrm{~s}$ & 76,13 \\
\hline
\end{tabular}

Fonte: Autores

\section{CONSIDERAÇÕES FINAIS}

Neste artigo se pode perceber a grande importância do processo de calibração de instrumentos, utilizado de todos os equipamentos devidos para aplicar a metodologia do processo de calibração automatizada.

Deste modo, o trabalho foi acreditado e normatizado, através do Certificado de Calibração CA1 - 116/201, emitido pelo Centro de Pesquisas de Energia Elétrica - CEPEL sob o título: "Calibração de Calibrador Digital nas escalas de corrente contínua e corrente alternada".

DLA (Departamento de Laboratórios de Adrianópolis), Pela RBC (Rede Brasileira de Calibração), sob o número de identificação 0008.

Sendo assim, acredita-se que atingiu os objetivos, em função da redução do tempo das aplicações na calibração, melhorou a segurança, visto que se trata de equipamentos elétricos, manteve a confiabilidade em relação ao processo de calibração manual, estando o método analisado de acordo com os requisitos da Norma NBR ISSO/IEC 17025:2005.

O Sistema foi analisado ao longo das atividades de calibrações automatizadas e manuais, desta maneira tornou-se possível averiguar a confiabilidade do sistema em todas as escalas propostas. No sistema adotou-se a calibração manual como referência, possibilitando calcular o erro normalizado, no qual aponta a compatibilidade de medidas de calibrações diferentes onde seu modulo é menor ou igual a 1 . Assim, o erro normalizado foi obtido o valor menor que 0,7 
estando dentro do limite estipulado, e garantindo a consistência dos valores conquistados. 0 presente trabalho obteve melhoria em relação ao tempo de calibração onde teve redução real do tempo de aplicação da metodologia. Na aplicação do processo geral observa-se redução de 76, $13 \%$ do tempo geral demandado.

No caso da metodologia para estimativa de incerteza de medição continuou atendendo todos os requisitos, afirmando o grau de confiabilidade dos processos de medições em torno de $96 \%$.

Notou-se a diminuição da vulnerabilidade a erros de cálculos e digitações, uma vez que trata se de dados que são inseridos diretamente na planilha através do software.

Sendo assim, aplicando os instrumentos envolvidos no processo de calibração automática permitiu monitorar a rotina de calibração nas escalas de tensão do multímetro Hewlett Packard, modelo 3458A, onde observou-se melhoria na otimização do tempo de calibração tornando-o método mais eficiente, garantiu a melhoria da qualidade dos resultados dos cálculos de incerteza de medição e diminuiu a probabilidade de erro humano.

\section{REFERÊNCIAS}

COUTO, P. R. G.; MONTEIRO, L. C. A Função de um laboratório de metrologia de acordo com o contexto de globalização. Duque de Caxias: [s.n.], 2000.

DINIZ, A. C. G. C.; MULLER, G. Entendendo a Norma ABNT ISO/IEC 17025: 2005. [S.I.]: [s.n.], 2007.

FLUKE. Fluke Calibração. Manual de instrução, 1989. Disponivel em:

$<$ https://br.flukecal.com/products/obsolete-products/5700a5720a-multifunction-

calibrators?quicktabs_product_details=4>. Acesso em: 14 jun. 2019.

INMETRO, I. N. D. M. N. E. Q. I. Expressão de incerterza de medição na calibração. Rio de janeiro: [s.n.], 1999.

INMETRO, V. VOCABULÁRIO INTERNACIONAL DE METROLOGIA Conceitos Fundamentais e Gerais e Termos associados. 3a. ed. [S.I.]: [s.n.], 2003.

INSTITUTO NACIONAL DE METROLOGIA, N. E. Q. I. (. Expressão da Incerteza de Medição na Calibração. Rio de Janeiro: Versão Brasileira do documento referência EA-4/02, 1999.

ISO, G. Guia para a Expressão da Incerteza de Medição. 3ạ edição Brasileira. ed. [S.I.]: [s.n.], v. INMETRO, ABNT, 2003. 